\title{
Frontières
}

\section{Traumatisme craniocérébral}

De la souffrance à la résilience

\section{Hélène Lefebvre et Marie-Josée Levert}

Volume 17, numéro 2, printemps 2005

Surtout, ne pas souffrir

URI : https://id.erudit.org/iderudit/1073493ar

DOI : https://doi.org/10.7202/1073493ar

Aller au sommaire du numéro

\section{Éditeur(s)}

Université du Québec à Montréal

ISSN

1180-3479 (imprimé)

1916-0976 (numérique)

Découvrir la revue

Citer cet article

Lefebvre, H. \& Levert, M.-J. (2005). Traumatisme craniocérébral : de la souffrance à la résilience. Frontières, 17(2), 77-85.

https://doi.org/10.7202/1073493ar
Résumé de l'article

Le traumatisme craniocérébral est une expérience déchirante pour les familles, mais aussi pour les professionnels de la santé qui vivent de l'impuissance face à la souffrance de la personne ayant le traumatisme craniocérébral et de ses proches. Le processus de deuil de la personne telle qu'elle était avant le traumatisme crânien et celui de leur vie antérieure est certes souffrant, mais s'accompagne d'un potentiel d'apprentissage et de croissance, tant pour les familles que les professionnels de la santé impliqués auprès d'elles. Une relation reposant sur le partage réciproque des savoirs et des expertises dans un rapport égalitaire est garante d'une relation satisfaisante, malgré une atmosphère chargée d'émotions. Le partenariat constitue en effet une stratégie intéressante qui permet de faire de ce moment de souffrance une expérience positive pour les familles et les professionnels de la santé. 


\section{Résumé}

Le traumatisme craniocérébral est une expérience déchirante pour les familles, mais aussi pour les professionnels de la santé qui vivent de l'impuissance face à la souffrance de la personne ayant le traumatisme craniocérébral et de ses proches. Le processus de deuil de la personne telle qu'elle était avant le traumatisme crânien et celui de leur vie antérieure est certes souffrant, mais s'accompagne d'un potentiel d'apprentissage et de croissance, tant pour les familles que les professionnels de la santé impliqués auprès d'elles. Une relation reposant sur le partage réciproque des savoirs et des expertises dans un rapport égalitaire est garante d'une relation satisfaisante, malgré une atmosphère chargée d'émotions. Le partenariat constitue en effet une stratégie intéressante qui permet de faire de ce moment de souffrance une expérience positive pour les familles et les professionnels de la santé.

Mots clés: deuil - adaptation résilience - intervention familiale relation familles-professionnels partenariat - traumatisme craniocérébral.

\section{Abstract}

Craniocerebral trauma is a devastating experience for families; it is equally so for the health professionals confronted with their own impotence in the face of the suffering of both the individual affected by the craniocerebral trauma and those close to that person. The mourning process of the state of being before the accident, their life before the accident is without a doubt difficult, and yet it also comes with the potential to learn and grow, as much for the families as the health professionals closely implicated with them. A relationship built on mutual sharing of knowledge and expertise in the spirit of true equality is the hallmark of a healthy relationship though it is highly charged with emotion. Partnership emerges as an ideal strategy to transform this situation of suffering into a positive experience for both members of the family and the involved health professionals.

Keywords: Mourning - adaptation - resilience - family intervention family-professional relationship partnership - craniocerebral trauma.

\section{TRAUMATISME CRANIOCÉRÉBRAL DE LA SOUFFRANCE À LA RÉSILIENCE}

Hélène Lefebvre, Ph. D.,

Faculté des sciences infirmières, Université de Montréal,

\section{Marie Josée Levert,} coordonnatrice de recherche,

Faculté des sciences infirmières, Université de Montréal.

Le deuil est un phénomène habituellement vécu par les personnes lors de la mort d'un être cher. Il existe toutefois d'autres situations de perte qui peuvent se révéler tout aussi difficiles à vivre et impliquent un processus de deuil: la perte de l'identité, la perte d'utilisation d'un membre ou d'un sens comme la vision ou l'audition, l'incapacité de réaliser certaines ambitions; la perte d'autonomie, un déménagement, la perte d'un emploi, une séparation ou un divorce, le départ d'un enfant de la maison, la retraite, la maladie, la mort d'un animal favori (Rando, 1986; Shanfield et Swain, 1984; Raphaël, 1983). Lorsqu'un événement semblable survient de façon soudaine et inattendue, la souffrance n'en est que plus intense. Le traumatisme craniocérébral (TCC) causé par un accident se situe dans la lignée de ces situations imprévisibles et soudaines qui ébranlent chaque année des milliers de familles canadiennes et québécoises (Statistique Canada, 1999; SAAQ, 2001).

L'avancement des technologies médicales de pointe et la rapidité avec laquelle les soins d'urgence sont offerts augmentent l'espérance de vie des personnes ayant subi un TCC (Yanko et Mitcho, 2001; Sampalis, Denis, Fréchette, Brown, Fleiszer, Mulder, 1997; Sampalis, Lavoie, Boukas et al., 1995). Apprendre qu'un proche a été victime d'un accident de la route et qu'il a subi un traumatisme crânien est une situation déchirante pour les familles, mais aussi pour les professionnels de la santé qui vivent un malaise et de l'impuissance devant la souffrance d'un proche. Celui-ci doit apprendre à vivre avec les incapacités physiques, cognitives et affectives qui vont affecter son fonctionnement et sa dynamique pour de nombreuses années, sinon toute la vie (Lefebvre, Pelchat, Swaine, Gélinas et Levert, 2004; Lefebvre, Vanier et al., 2004; Frénisy, Béhony, Freysz, Honnart, Didier et Sautreaux, 1999; Gélinas, 1998; Shaw, Chan et Lam, 1997; Leach, Frank, Bouman et Farmer, 1994; Jacobs, 1988). L'expérience de ces familles est d'autant plus difficile à vivre que le TCC est une situation de santé pour laquelle le pronostic est longtemps incertain, la réadaptation complexe, et qui résulte en l'apparition progressive d'incapacités, de problèmes médicaux, sociaux et humains, avec souvent la perte de l'espoir et une souffrance de vivre.

Quelques études récentes et l'expérience clinique auprès de personnes ayant subi un traumatisme craniocérébral et de leurs familles sont à l'origine de cette réflexion.

\section{SOUFFRANCE DES FAMILLES}

Lucie, 21 ans, sort d'une discothèque; elle roule vite sur l'autoroute. Il pleut légèrement. Le téléphone sonne au milieu de la nuit:«Ici l'urgence de l'hôpital X, vous avez une fille qui se prénomme Lucie...». L’univers bascule. Les témoignages entendus se ressemblent étrangement: l'incertitude du moment, le choc, l'incompréhension, le 
vide, l'irréparable, cet appel téléphonique qui sépare irrémédiablement la vie entre un avant et un après. Les proches se précipitent à l'hôpital. Ils voudraient savoir, voir, toucher, prendre la personne dans leurs bras, agir, faire quelque chose... C'est l'incertitude, l'attente insoutenable. Pourquoi? Comment? Que va-t-il arriver?

Lucie est dans le coma à l'unité d'urgence, dans la salle de réanimation. Dans le monde mystérieux de l'urgence, l'équipe de spécialistes s'affaire derrière la porte close. Réanimation veut dire faire revenir à la vie. Cependant, chacun sait que parfois c'est l'irrémédiable, c'est là que le destin bascule, la vie s'est enfuie. Parfois elle est là, mais la personne est inerte, endormie, dans le coma. Ce coma est source d'une incertitude invivable. Les proches, derrière la porte, imaginent le pire.

La personne blessée comateuse, inerte, détermine toute l'ambiance des soins, par sa présence-absence et sa dépendance. Elle n'a pas de besoins autres qu'objectifs, elle ne répond pas, elle est absente (Cohadon, 2000). La personne apparaît ainsi pour la famille et les professionnels de la santé chargés de lui prodiguer les soins. Le coma instaure une situation singulière. Le corps est présent, absent de communication, sans conscience du monde extérieur. Sa vie dépend de chaque élément technique mobilisé autour de lui. La personne comateuse ne souffre pas du coma, c'est la famille qui en souffre et les professionnels susceptibles d'être confrontés à la mort ou au non-éveil, à l'échec (Cohadon, 2000).

L'impossibilité de relation avec la personne et la perte de son autonomie la placent dans une position d'objet. Elle est dépersonnalisée. Il n'y a plus de repères de singularité, de l'individualité, ni repère dans l'espace et le temps. Dès son entrée à l'urgence, la personne est vouée à un statut d'objet, respectée certes par les professionnels de la santé, mais le caractère de haute technologie des soins fait en sorte qu'ils décident et exécutent ce qui est nécessaire sans atermoiement. Cette situation protège contre toutes formes d'angoisse. Selon Cohadon (2000), la personne comateuse est perçue comme un objet parce que si elle était un sujet, elle renverrait en miroir aux individus qui l'entourent une image d'eux-mêmes qu'ils ne peuvent regarder, une représentation de leur propre fragilité, de leur propre mort. De plus, la personne comateuse est objet parce qu'elle est soumise à la science et à la technique comme seul un objet peut l'être. L'angoisse de chacun est palpable. L'assurance technique des professionnels de la santé pour appliquer toute l'efficacité de la science semble le seul rempart contre la possibilité de mort de la personne et l'angoisse qu'elle suscite (Cohadon, 2000).
La personne blessée entre ainsi dans la filière de soins. De l'urgence, elle ira aux soins intensifs, puis à l'unité spécialisée avant d'aborder la réadaptation fonctionnelle puis, peut-être, un retour dans la société.

Aux soins intensifs, les familles affirment que l'information transmise au comptegouttes nuit à une bonne intégration de l'événement, rend l'attente difficile à vivre et entrave leur processus de deuil (Lefebvre, Pelchat et Levert, à paraître; Lefebvre, Pelchat et al., 2004; Brzustowski, 2001; Bolduc, 1996). Pour les médecins et les professionnels des soins aigus, le manque d'information évoqué par les familles est intimement lié à l'état de choc psychologique dans lequel elles se trouvent (Fallowfield et Jenkins, 2004; Lefebvre, Pelchat et al., 2004; Ptacek et Ellison, 2000; Maguire, 1998). Cependant, le manque d'information donnée à la famille dans le processus de l'annonce semble aussi associé au fait que les médecins et les professionnels de la santé vivent eux-mêmes beaucoup d'incertitude à cause de la condition clinique évolutive du TCC: «On ne peut pas les aider dans leur incertitude parce que leur incertitude est aussi notre incertitude» (Lefebvre, Pelchat et al., 2004). Confrontés à de nombreux éléments inconnus sur le plan du pronostic, les médecins et professionnels de toutes les phases de soins sont nombreux à se dire hantés par la «peur de se tromper», par la crainte d'ébranler davantage la famille ou de lui donner de faux espoirs: "Pour ne pas donner un trop grand coup à la famille. Parce que dire à un membre de la famille: "Bien, je ne crois pas que votre fils va retourner vivre à la maison" ou "Je ne crois pas qu'il pourra retourner travailler ou à l'école", c'est une grosse pilule à avaler.» Cette situation, où les incertitudes sont nombreuses, affecte le contenu de l'information qui est communiquée et rend plusieurs médecins mal à l'aise dans leurs relations avec les familles qui sont généralement avides de réponses. D'ailleurs, la plupart des personnes et des familles reconnaissent implicitement l'incertitude des médecins et des professionnels.

Chacun trouve comme il peut en luimême les ressources pour faire cette traversée. Il est essentiel dans cette situation que la famille reçoive du soutien des proches, des amis, des professionnels, des services (Lefebvre et al., à paraître; Frénisy et al., 1999; Rando, 1986; Doka, 1989). Rares sont les familles qui affirment avoir été aidées dans leur processus de deuil par les médecins et les professionnels, mais elles disent que l'équipe médicale a été parfaite, compétente (Lefebvre, Pelchat et al., 2004).

\section{LE PROCESSUS DE DEUIL}

Le processus de deuil est un mécanisme psychologique qui permet à la personne qui vit la perte de se réorganiser intérieurement afin de s'adapter à l'événement et de réorienter sa vie par la suite. Ce processus vise à permettre à la personne de se détacher affectivement de l'objet investi pour être capable ultérieurement de réinvestir de nouveaux attachements.

Kübler-Ross (1981) a décrit les étapes qu'une personne traverse habituellement à mesure qu'elle se rapproche de sa mort. Elle les a présentées dans l'ordre chronologique où les personnes les vivent habituellement: choc initial, déni, révolte, marchandage, tristesse, acceptation. Cependant, d'autres auteurs ont élaboré d'autres modèles répondant à des situations particulières.

Pelchat et Lefebvre (2004) ont développé un modèle du travail du deuil dans le cadre du Programme d'intervention familiale (PRIFAM), qui décrit les différentes phases du processus d'adaptation chez une personne en situation de stress intense.

\section{Une conception dynamique,}

systémique et évolutive

L’originalité de ce modèle du deuil réside dans son caractère dynamique et systémique, de même que dans sa conception évolutive. D'un point de vue dynamique, ce modèle considère l'altérité des membres d'une famille, soit la singularité de leur fonctionnement psychologique, de leurs valeurs, de leurs croyances, de leur manière de réagir et de faire face au stress et de leur façon d'exprimer leur souffrance. Chacun des membres de la famille vit les phases d'une manière qui lui est propre et qui correspond à l'organisation de sa personnalité, à sa manière d'être en relation avec lui-même et avec le monde et selon la façon dont il a vécu antérieurement ses expériences de deuil, les deuils non résolus dans le passé risquant de refaire surface. Certaines personnes analysent rationnellement la situation alors que d'autres expriment davantage de colère et de tristesse. Ces différentes manières de vivre un deuil sont cependant en interinfluence. L'aspect systémique de ce modèle montre que les réactions des membres de la famille et leur cheminement dans le processus de deuil ont un impact sur le deuil des autres membres. Le caractère évolutif du modèle tient au fait qu'il existe un va-et-vient entre chacune des phases. Le cheminement des familles est constitué de périodes évolutives vers l'adaptation, mais aussi de périodes régressives, par exemple de l'adaptation à la dénégation à la suite de l'apparition d'une complication de l'état de santé de la personne ayant subi le TCC. Ces périodes de régression mènent à une nouvelle progression: dans les familles 


\section{SCHÉMA 1 - CARACTÈRE DYNAMIQUE ET ÉVOLUTIF DU PROCESSUS DE DEUIL}

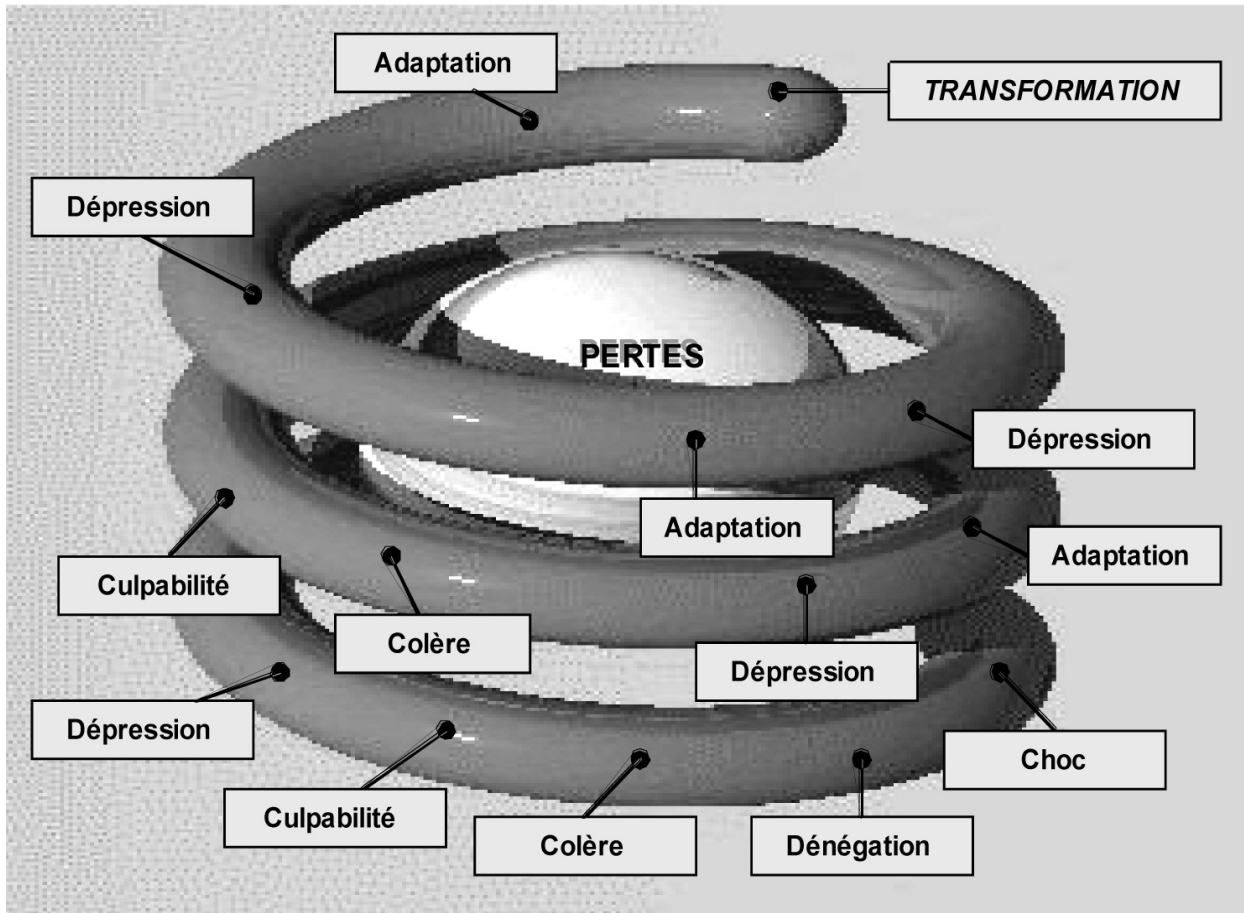

Schéma: Hélène Lefebvre, PH. D., et Marie-Josée Levert, Ph. D. (cand.), Équipe de recherche multidisciplinaire sur la famille (ÉRIFAM), Université de Montréal.

ayant de la difficulté à trouver des services adéquats, certaines personnes peuvent développer des qualités insoupçonnées, telle la débrouillardise, ou modifier leurs croyances et le sens qu'elles donnent à la vie. Le cheminement dans le processus de deuil suscite une transformation des membres de la famille, évolution qui fait en sorte qu'ils ne redeviennent jamais ceux qu'ils étaient avant. L'aspect systémique considère que cette transformation de chacun se répercute sur les autres et métamorphose l'ensemble de l'organisation familiale. Le schéma 1 présente les aspects dynamiques et évolutifs du processus de deuil.

Le modèle est constitué de six phases interreliées à travers lesquelles la personne endeuillée chemine: le choc, la dénégation et le déni, la colère, la culpabilité, la dépression, l'adaptation et la transformation. L'expérience clinique et la recherche ont permis d'enrichir ce modèle et d'y adjoindre une phase préparatoire au processus de deuil, la phase d'appréhension.

\section{L'appréhension}

\section{un appel au milieu de la nuit}

Le processus de deuil s'amorce habituellement avec l'annonce du diagnostic. Cependant, certaines situations comme l'appel téléphonique du centre hospitalier révélant qu'un membre de la famille a eu un accident, un infarctus ou lorsque la personne est en attente de diagnostic, semblent amorcer une phase préliminaire au pro- cessus de deuil. Au cours de cette phase, que l'on pourrait nommer phase d'appréhension, la famille ébauche des scénarios négatifs et positifs, vit de l'ambiguïté, de l'incertitude et ressent une large gamme d'émotions parfois contradictoires, dont une grande part d'anxiété.

L'accueil, l'attente, les premiers mots: Est-ce qu'elle va vivre? Que peuvent dire les professionnels de la santé? Espérer, être prudent, différer le verdict, attendre les résultats de l'opération, attendre le résultat d'une tomodensitométrie, attendre vingt-quatre heures, les premiers jours... (Lefebvre et al., à paraître; Cohadon, 2000). La famille affronte seule, et le plus souvent avec dignité, tout le répertoire de la détresse humaine, malgré la sollicitude des professionnels de la santé. Elle vit de l'angoisse, du chagrin, de l'impuissance, une alternance d'espoir et de désespoir, confiance dans les professionnels de la santé, solitude, secours de la spiritualité (Lefebvre $e t$ al., à paraître; Bolduc, 1996; Paturau, 1984).

L'expérience clinique et la recherche montrent que cette phase semble préparer la famille au choc de l'annonce de l'événement.

\section{Le choc de l'annonce}

Puis survient la rencontre avec le médecin. Lindicible est dit, l'inaudible est entendu. L'annonce du diagnostic est un moment effroyable pour la famille qui est sous le choc de la nouvelle. Le fonction- nement psychologique de chacun des membres de la famille risque alors d'être débordé par l'impossible qui s'est produit, par l'événement qui dépasse leur capacité psychique d'y faire face. Cette annonce paralyse le fonctionnement psychique. L'annonce du TCC prend alors une valeur traumatique qui se manifeste par un état de stupeur et de sidération dont l'intensité varie en fonction de la manière dont les membres de la famille se représentent le traumatisme crânien et la situation, mais qui laissera une cicatrice indélébile.

\section{La dénégation:}

«Non, ce n'est pas vrai!»

L'état de stupeur laisse graduellement place à une montée de l'angoisse. Afin de s'en défendre, le psychisme met généralement en place le mécanisme de dénégation. La dénégation est un mécanisme psychique permettant de contrer l'angoisse qui s'installe graduellement lorsque le fonctionnement psychique reprend ses moyens devant l'insupportable situation: "Non, ce n'est pas vrai!» Selon l'organisation de la personnalité de l'individu ou lorsque le mécanisme de dénégation s'avère insuffisant pour contenir l'angoisse, un déni de la réalité peut se mettre en place. Ce mécanisme, plus massif et moins adaptatif que le précédent, conduit à un rejet de la réalité vécue comme insupportable, et à la construction d'une néo-réalité où le TCC n'existe pas. Il ne s'agit pas de penser que cela n'a pas pu se produire, mais plutôt de ne pas y penser du tout.

Ces mécanismes ont d'abord une fonction adaptative. Les circonstances et la gravité du coma déterminent en grande partie l'attitude des familles. Toutefois, la situation insupportable de l'accident qui fait basculer d'un seul coup la vie d'une personne très souvent jeune et l'état comateux dans lequel elle se trouve durant les premiers jours ou les premières semaines suivant l'accident ne laissent souvent d'autres ressources que la dénégation. Celle-ci protège les membres de la famille de leur souffrance et leur permet d'entrer graduellement en contact avec la situation pour commencer à l'assimiler en maintenant leur sentiment d'estime d'eux-mêmes, en réduisant l'angoisse et en générant l'espoir. La dénégation persistera longtemps au cours du réveil et même au-delà: «La famille tourne autour du corps du blessé endormi, avec ses électrodes, ses sondes et autres appareils en espérant qu'il s'éveille, qu'il lui fasse un signe qu'il n'est pas tout à fait absent.»C'est lui, «si fort, si travaillant, si affectueux». «Non, c'est impossible que cela lui soit arrivé à lui!». Cohadon (2000) et Doka (1989) indiquent que le fait de la présence physique de la personne, contrairement au deuil lors de la mort d'un 
être cher, et certains traits de personnalité font en sorte que la famille persistera plus longtemps dans la dénégation, se sentant incapable d'un certain détachement émotif à l'égard de la personne. La persistance dans l'utilisation de ces mécanismes risque toutefois de susciter des difficultés d'adaptation majeures, puisqu'elle ne permet pas la reconnaissance de la réalité de la situation et entrave la mise en œuvre d'autres stratégies permettant de s'y adapter.

\section{La colère:}

«Pourquoi n'as-tu pas fait attention?»

La diminution progressive du déni amène progressivement la famille à prendre conscience de la réalité. Cette prise de conscience s'exprime par des réactions de révolte, de colère envers la personne blessée. "Pourquoi conduisais-tu si vite? Je t'ai dit souvent que c'était dangereux. Tu savais que tu ne pouvais pas conduire en sortant de ce bar! Voistu la peine que tu nous causes?» La colère contre la personne ayant subi le traumatisme craniocérébral est généralement impossible à exprimer. Elle se dirigera alors vers d'autres cibles, les ambulanciers qui ne sont pas intervenus assez vite, le personnel soignant qui ne dit rien, d'autres membres de la famille.

\section{La culpabilité:}

«Jamais je n'aurais dû la laisser partir... »

La colère contre la personne se tourne peu à peu vers le proche qui se sent responsable de l'accident, alors considéré comme un reproche vivant. Toutes les relations familiales, surtout celles entretenues avec la personne accidentée, se rejouent. Attachement, détachement, compromis, mensonges, tout est repassé et de là surgit la déchirure, l'angoisse, la culpabilité. Coupable de ne pas avoir été là, coupable de l'avoir laissé partir, coupable de ne pas avoir assez aimé, coupable... mais il est trop tard pour ce qui n'a pas été dit, pour faire ce qui n'a pas été fait.

Ces sentiments risquent d'entraîner la surprotection excessive des proches à l'égard de la personne ayant subi le TCC. Cela se manifeste la plupart du temps au moment du transfert en réadaptation alors que la personne ayant le traumatisme craniocérébral doit apprendre à redevenir autonome. La famille protège la personne des risques d'accident par exemple en faisant des tâches quotidiennes à sa place, en contrôlant chacune de ses activités.

\section{La dépression:}

\section{un processus d'introspection}

La colère et la culpabilité permettent graduellement au proche d'exprimer sa tristesse, de se centrer sur son monde intérieur et d'identifier les émotions suscitées par la situation. Cette période dépressive, loin de la dépression chronique où le fonctionnement est paralysé, marque le début de l'intégration de la situation engendrée par les changements physiques, psychologiques et comportementaux apportés par le traumatisme craniocérébral. C'est une période constructive qui amène le proche à réfléchir sur sa vie, à vivre sa tristesse sur une période plus ou moins longue. Les espoirs, les désirs, les attentes, les projets sont remis en scène avec une grande intensité. Ce regard posé vers l'intérieur permet aussi au proche de réfléchir sur le TCC et sur les multiples émotions que la situation suscite en lui, même s'il est généralement difficile d'en prévoir l'issue. C'est cette période dépressive qui permet au proche d'exprimer ses conflits et ses émotions de manière moins "déguisée» et de résoudre son deuil de la personne telle qu'elle était avant l'accident.

\section{L'adaptation:}

apprendre à vivre avec la différence

Après une très longue période, le processus de deuil amène la famille à s'adapter à la nouvelle personne qu'est devenu le conjoint, la mère, le père, la sœur ou l'ami. Le proche apprend à vivre avec les incapacités résiduelles, avec les limites et l'impact qu'elles ont sur la vie familiale et sociale. Il apprend à reconnaître les compétences de la personne. Tranquillement, la famille réinvestit la nouvelle personne.

\section{La transformation: réinventer sa vie}

Pour la plupart des théoriciens du deuil, le processus prend fin avec l'acceptation de la perte. La plupart s'entendent pour affirmer que l'acceptation constitue en fait une adaptation, une résignation, une réorganisation ou un rétablissement, selon le cas. Cette conception fait toutefois abstraction du changement qui s'opère au sein de chacun des individus et qui se répercute sur l'ensemble de la famille. Les travaux sur l'adaptation des familles effectués par l'Équipe de recherche en intervention familiale (ÉRIFAM) dans le cadre du Programme d'intervention familiale ont démontré que le travail du deuil introduit les personnes et les familles dans un processus d'apprentissage qui les transforme. Ces réflexions ont permis de distinguer une nouvelle phase au processus de deuil qui constitue son aboutissement: la phase de transformation. L'adaptation/transformation est la capacité de la famille à se sentir compétente et confiante pour utiliser ses ressources et son savoir-faire dans la gestion de son quotidien, pour s'autodéterminer et actualiser ses nouveaux objectifs de vie (Pelchat et Lefebvre, 2004, p. 77).

Le processus de deuil est certes souffrant, mais il comporte un potentiel d'apprentis- sage et de croissance. Lors de la survenue du TCC chez un membre de la famille, celle-ci puisera en elle les forces intérieures et les ressources pour faire face à l'épreuve. L'Équipe de recherche ÉRIFAM considère qu'au-delà de la capacité à exprimer ses émotions et à apprendre à vivre avec la nouvelle réalité pour établir de nouveaux attachements, le processus de deuil est enrichi d'un processus d'apprentissage qui amène les familles à se transformer.

La transformation réfère à un changement qui émane de l'intérieur au plan des valeurs, des croyances et des connaissances. Ainsi, les familles acquièrent un nouveau savoir, développent de nouvelles compétences, prennent conscience de l'importance de l'entraide et du partage des ressources et accueillent graduellement dans sa différence la personne ayant subi le traumatisme craniocérébral. Ces nouveaux savoirs émanent de l'expérience du processus de deuil imposé par la trajectoire de la personne. Ainsi, les familles ne font pas que s'adapter, elles transforment leur expérience en de nouveaux projets de vie dans toutes les sphères de leur existence.

\section{La récurrence}

Lorsqu'il s'agit de problèmes de santé chroniques comme ceux liés au traumatisme craniocérébral, le processus de deuil n'est jamais achevé. Le caractère récurrent du processus se rapproche des concepts de deuil ambigu (ambigous loss) [Boss, 1999] ou non affranchi (St-Charles, 1998) [disenfranchised grief, Doka, 1989]. Le deuil ambigu est lié à une situation où les faits ne sont pas clairement décrits ou ne sont pas disponibles, le diagnostic incertain et le pronostic impossible à établir rapidement et où les informations varient d'un professionnel à l'autre ou encore les nouvelles sont parfois bonnes, parfois mauvaises ou inversement. Il est alors impossible pour les membres de la famille de donner une signification commune à l'événement; comme, par exemple, face à la naissance prématurée d'un enfant, la maladie d'Alzheimer, le sida, une maladie ou des incapacités physiques ou psychologiques. Le deuil non affranchi représente quant à lui l'expérience des personnes endeuillées dont les droits, les rôles et la capacité d'être en deuil ne sont pas reconnus socialement (St-Charles, 1998; Doka, 1989). Cette invalidation peut être liée soit à la relation avec l'objet de la perte, qui ne peut pas être vécue ouvertement et n'est pas soutenue par l'entourage parce que la relation avec la personne n'est pas acceptée socialement (ex.: homosexualité), soit parce que l'objet de la perte est invalidé (ex.: avortement) ou lorsque les capacités de la personne à vivre un deuil ne sont pas reconnues (ex.: personne présentant 


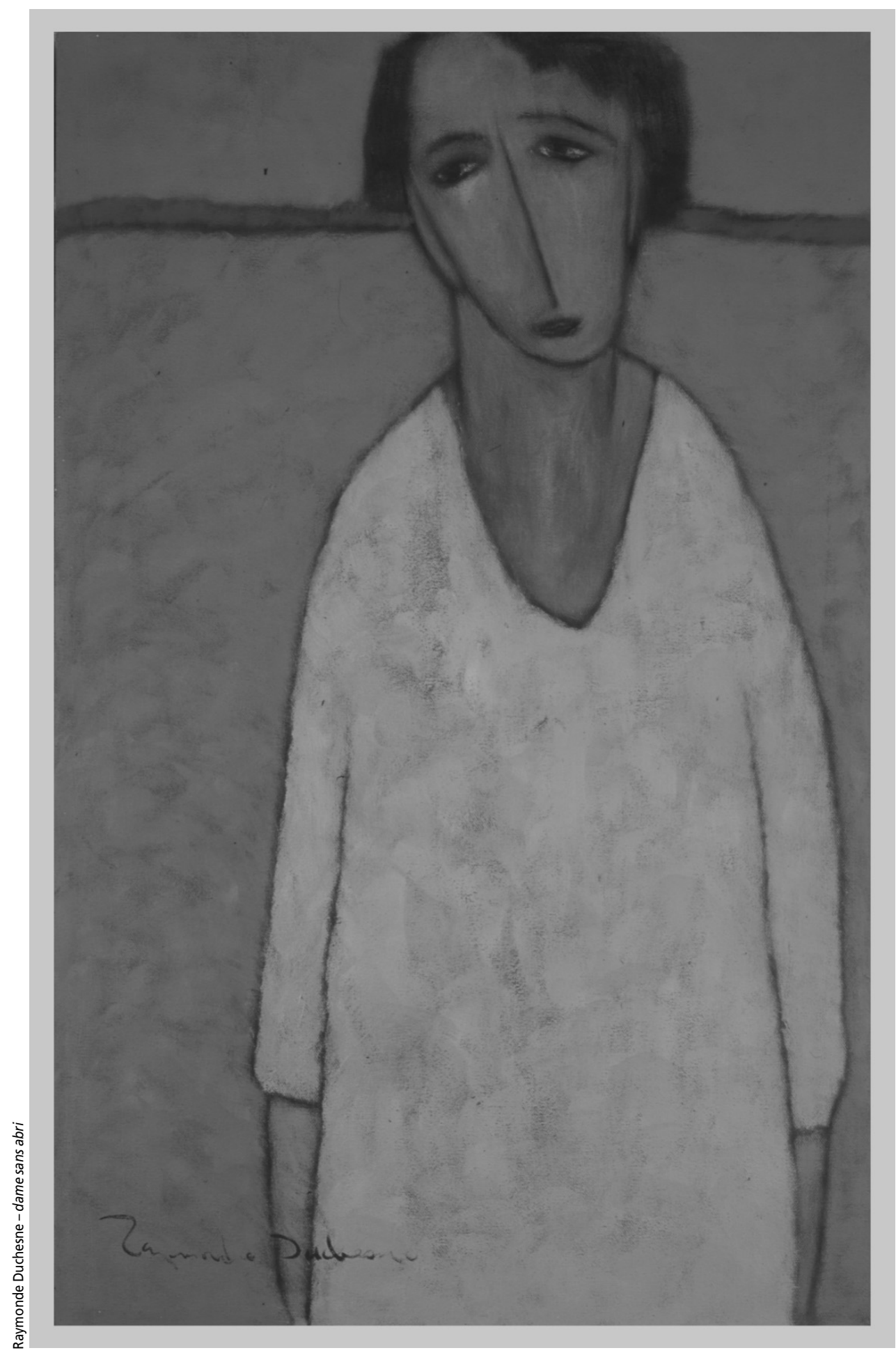

une déficience intellectuelle). Doka (1989) propose le concept de deuil psychosocial dans les situations de santé où il y a une perte psychocognitive, tel un changement de personnalité, et explique que la famille devra faire face à la mort de l'être psychosocial du proche atteint par la maladie ou les incapacités.

Ces concepts de deuil non affranchi et deuil psychosocial apportés par Doka (1989) sont particulièrement intéressants, car ils mettent en contexte le processus de deuil dans un cadre social. Ces expériences

\section{l'errance pleurante le clochard guettant un morceau de plaisir}

à la fois d'être ambigu, en raison de la complexité du tableau clinique qui suscite tant d'incertitudes, et non affranchi puisque les proches seront sans cesse confrontés aux incapacités, aux complications médicales ou au regard des autres.

Quelle que soit la manière dont le deuil est vécu, il a un impact significatif sur la personne ayant le TCC et sa famille et sur leur adaptation à l'événement, sur l'accomplissement des tâches adaptatives (Brzustowski, 2001; Pelchat, Lefebvre et Bouchard, 2001; Nolte, 2000; Pelchat, Lefebvre, Bouchard, Kalubi, Michallet et Rousseau, 2000; Ptacek et Allison, 2000; Denormandie et Hirsch, 1999; Frénisy et al., 1999; Campbell et Sanson-Fisher, 1998; Bolduc, 1996; Cohen, 1993).

\section{LES TÂCHES ADAPTATIVES}

Le TCC engendre un stress important dans toutes les sphères de la vie des personnes l'ayant subi et des membres de la famille. D'ailleurs, nombre d'études ont déjà dévoilé l'impact systémique de cette problématique de santé (Frénisy et al., 1999; Saint-Charles, 1998; Krier, 1997; Madinier, 1997 ; Blaise, 1997; Schumacker et Meleis, 1995). C'est un événement qui perturbe l'équilibre familial et plonge la famille dans une transition situationnelle pour laquelle elle n'est pas préparée: incapacités potentielles et graduelles, besoins de la personne ayant subi un TCC, responsabilités supplémentaires et nouvelles. La situation de santé et l'avenir de la personne affectée étant incertains, la famille devra composer avec cette nouvelle réalité et redéfinir sa perception de la situation en tenant compte des séquelles, des capacités résiduelles, mais aussi des capacités réelles de la personne (Lacroix et Assal, 2003; Ergh, Hanks, Rapport et Coleman, 2003; Brzustowski, 2001; Dell Orto et Power, 2000; Koskinen, 1998; Ergh, Krier, 1997; Madinier, 1997 ; Cohen, 1993).

Dès les premiers instants suivant l'accident, la famille a à redéfinir le rôle de chacun de ses membres. Elle doit d'abord pallier l'absence de la personne qui a subi le TCC. Le soutien financier, l'aide aux devoirs pour les enfants, la préparation des repas et les autres rôles informels de la personne doivent être pris en charge par un autre 
membre de la famille. Au fur et à mesure que les séquelles et incapacités se précisent, toute la dynamique familiale devra être réorganisée en tenant compte des nouvelles limites et des capacités de la personne (Dell Orto et Power, 2000; Krier, 1997). La situation familiale peut rarement revenir à ce qu'elle était avant le TCC. Cependant, les membres de la famille peuvent arriver à reconstruire une dynamique relationnelle qui soit satisfaisante pour tous (Dell Orto et Power, 2000). Pour ce faire, la famille doit vivre le deuil de l'identité de la personne pour s'adapter à la nouvelle réalité (Krier, 1997; Madinier, 1997; Pelchat, 1989, 1995). La personne, telle qu'elle a été connue et investie par les membres de la famille avant l'accident, n'est souvent plus ce qu'elle était. Ce changement implique que chacun désinvestisse ou renonce à cette image antérieure pour être en mesure de s'attacher à la personne telle qu'elle est après le TCC. Les études rapportent une évaluation relativement positive du fonctionnement familial pour les proches (Ergh, Rapport, Coleman et Hanks, 2002). Cependant, l'intensité du fardeau subjectif, de détresse, de poids ou surcharge (strain) est généralement de modérée à extrême. Ce phénomène s'explique par le fait que les membres de la famille s'adaptent, réajustent leurs attentes face à la personne ayant subi le TCC et arrivent à être relativement satisfaits de leur fonctionnement familial malgré le sentiment de fardeau élevé.

Tout au long de ce processus, les membres de la famille doivent également préserver entre eux et avec les autres personnes significatives de leur entourage des relations qui soient satisfaisantes. La situation du TCC, par le stress qu'elle impose sur la dynamique familiale, peut faire ressurgir des conflits latents ou anciens entre ses membres (Frénisy et al., 1999; Madinier, 1997). De même, cet événement amène la famille à se centrer avec beaucoup d'intensité sur le membre qui a subi un TCC. Le monde extérieur, les loisirs, les amis, le travail et la famille élargie, risquent alors d'être désinvestis, mis à l'écart (Dell Orto et Power, 2000). En effet, les études montrent que les personnes ayant un TCC et leurs proches forment un groupe socialement isolé (Colantonio, Ratcliff, Chase, Kelsey, Escobar et Vernich, 2004; Lefebvre, Pelchat et al., 2004; Lefebvre, Vanier et al., 2004; Dawson et Chipman, 1995). Selon Blaise (1997), ce mouvement de repli, destructeur à long terme, est envenimé par la société qui en vient à traiter la personne ayant subi le TCC et la famille «comme s'ils n'étaient qu'un». En effet, il semble que le fardeau vécu par les proches à la suite du TCC soit davantage relié aux séquelles comportementales et affectives de la personne qu'aux incapacités physiques et cognitives (Ergh et al., 2003). En effet, ce type de séquelles entrave le processus d'adaptation puisqu'au plan physique ces personnes ont souvent une apparence normale et que les incapacités, généralement subtiles, sont peu évidentes. Il est souvent difficile pour les amis, les collègues de travail et la population en général de faire face à l'agressivité ou à des comportements qui semblent socialement mésadaptés. La sauvegarde des relations avec les proches et avec les professionnels de la santé permet à la famille de se prémunir contre l'isolement, d'élargir le champ de ses ressources externes afin de s'adapter et d'aller de l'avant (Perlesz, Kinsella et Crowe, 1999).

L'ensemble de ce processus débute dès les premiers instants de l'hospitalisation et se poursuit bien après la fin de la réadaptation. Les familles doivent trouver en elles et autour d'elles les ressources nécessaires pour y faire face. Elles sont engagées dans une lutte pour survivre qui mobilise toutes leurs énergies, mais qui leur permet aussi de découvrir leur potentiel de vie (Cyrulnik, 2002; Brzustowski, 2001; Fondation pour l'enfance, 2001; Fischer, 1994). $\mathrm{Vu}$ la rareté des moyens dans le contexte économique contemporain, toute crise vécue par les familles risque de perdurer et d'hypothéquer les possibilités de mener une vie paisible (Cyrulnick, 2002; Pelchat et Lefebvre, 2002; Fondation pour l'enfance, 2001; Fisher, 1994). Ces constats montrent que le rétablissement de l'équilibre familial peut nécessiter davantage de ressources à long terme, tel le soutien des professionnels de la santé.

\section{SOUFFRANCE DES PROFESSIONNELS}

Le TCC est une situation de santé qui suscite beaucoup d'angoisse et d'incertitude pour les familles (Ptacek et Allison, 2000; Frénisy et al., 1999; Kalubi, Michallet, Bouchard et Pelchat, 1998; Bolduc, 1996) mais aussi pour les professionnels de la santé qui se sentent souvent impuissants devant leur souffrance inconsolable (Lefebvre, Pelchat et al., 2004; Pelchat et al., 2000). Dès les premiers moments suivant l'accident, les médecins et les professionnels ressentent un malaise à annoncer un traumatisme crânien, à parler de son évolution et des incapacités qui risquent de s'installer avec le temps, comme de faire face aux réactions des proches, d'autant plus que celles-ci sont généralement difficiles à prévoir (Fallowfield et Jenkins, 2004; Brzustowski, 2001; Denormandie et Hirsch, 1999; Maguire, 1998; Saint-Charles, 1998; Brière, 1996; Cohen, 1993).

La situation de l'annonce place le médecin et les professionnels de la santé, dans le rôle du «porteur de mauvaise nouvelle»; eux qui sont riche de leur savoir disciplinaire pour guérir. Ce constat, jumelé à l'impact affectif de l'annonce sur l'ensemble du système familial, alourdit la tâche des médecins et des professionnels de la santé. Ceux-ci se sentent généralement impuissants à contenir les réactions de désespoir, de chagrin ou de colère, qui ont un impact dommageable sur la santé psychologique de la personne et des membres de sa famille (Fallowfield et Jenkins, 2004; Maguire, 1998). En effet, les professionnels ont parfois de la difficulté à faire face à des réactions qu'ils n'avaient pas anticipées. Le fait d'entreprendre un dialogue centré sur les émotions risque aussi de soulever la question du pronostic que les médecins et les professionnels préfèrent parfois éviter (Fallowfield et Jenkins, 2004; Maguire, 1998). En effet, il leur est difficile de prévoir l'évolution à court, moyen et long terme de l'état physique, cognitif et affectif de la personne qui a subi un TCC (Lefebvre, Pelchat, Kalubi et Michallet, 2002; Pelchat et al., 2000; Denormandie et Hirsch, 1999; Mishel, 1997 ; Bolduc, 1996; Cohen, 1993). En somme, les médecins et les professionnels ont généralement le sentiment de ne pas être suffisamment outillés pour effectuer cette tâche (Fallowfield et Jenkins, 2004).

Les recherches montrent que, face à ce malaise, les professionnels ont trop souvent tendance à se désengager de la relation (Fallowfield et Jenkins, 2004; Denormandie et Hirsch, 1999; Maguire, 1998). Ils adoptent alors des comportements comme se retirer de la relation; laisser la famille à elle-même après leur avoir présenté l'information; fuir sous des prétextes organisationnels; ou encore, se détacher affectivement de la situation.

Comme l'ont déjà montré plusieurs auteurs, cette situation nuit à l'adaptation individuelle et familiale (Brzustowski, 2001; Nolte, 2000, Pelchat et al., 2000; Ptacek et Allison, 2000; Denormandie et Hirsch, 1999; Campbell et Sanson-Fisher, 1998; Bolduc, 1996; Cohen, 1993). En

LES FAMILLES ET LES PROFESSIONNELS VIVENT, CHACUN DE LEUR CÔTÉ, DES SENTIMENTS D'IMPUISSANCE ET DE PERTE DE CONTRÔLE 


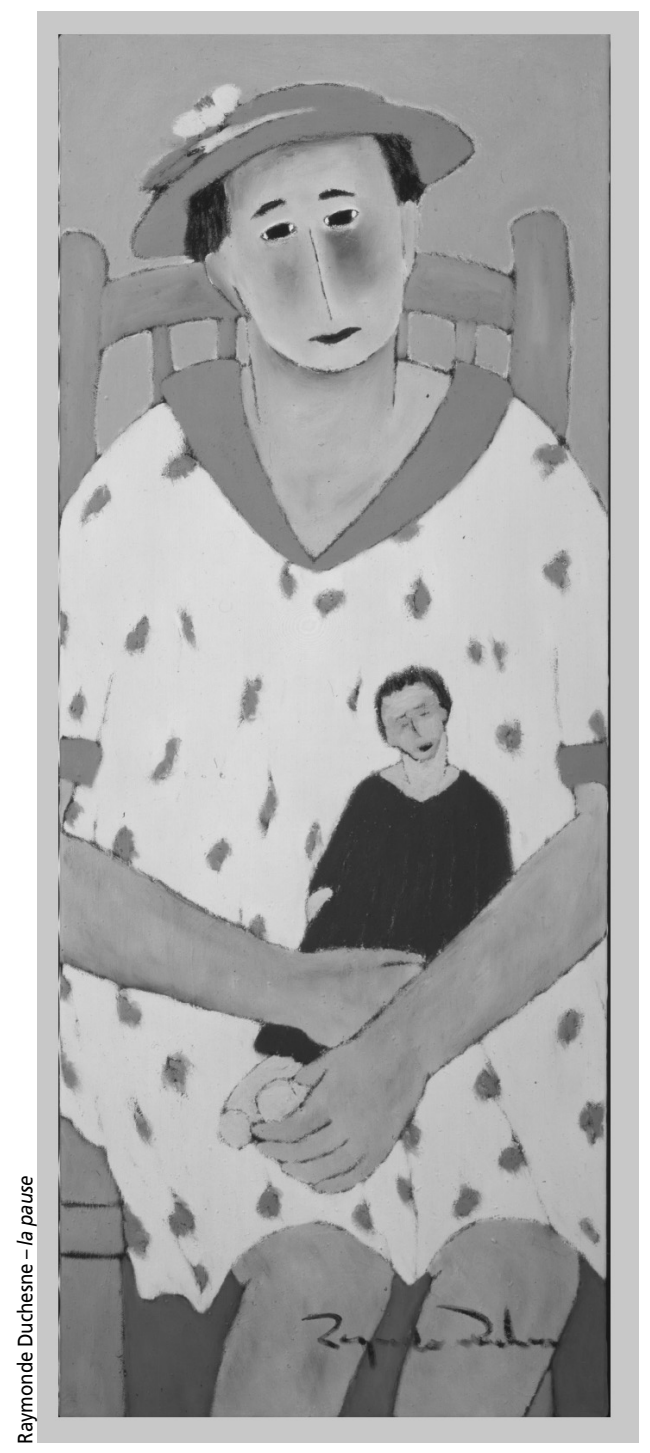

effet, les auteurs s'entendent sur le constat que l'expérience d'incertitude relative au TCC et la difficulté à faire face aux réactions individuelles et familiales ont un impact direct sur la qualité des relations famillesprofessionnels et, par le fait même, sur le processus d'adaptation à la nouvelle situation individuelle et familiale. Dans le contexte de santé actuel, qui insiste sur le soutien des habiletés d'adaptation des familles et leur responsabilisation des soins à donner à la personne qui a le problème de santé, cette réalité ne peut être négligée (Bouchard, Pelchat et Boudreault, 1995).

\section{DE LA SOUFFRANCE À LA RÉSILIENCE}

Le bilan des recherches, l'expérience clinique et les témoignages des patients, des familles, des médecins et des professionnels de la santé démontrent que deux mondes de perceptions et deux visions différentes se rencontrent (Lefebvre et al., à paraître; Pelchat et al., 2000; Denormandie et Hirsch, 1999; Girgis, Sanson-Fisher et Schofield, 1999). Les deuils sont des étapes de la vie

\section{à trembler devant la vie}

\section{au ralenti je la respire}

qui permettent de découvrir de nouvelles forces, peut-être inconnues jusqu'alors. Grâce à ces expériences, la perception de la vie se modifie et transforme chacun des acteurs (Man, 2002; Pelchat et al., 2001).

C'est d'ailleurs sur ce constat que s'appuie le concept de résilience. La résilience est définie comme la capacité de chacun des membres de la famille à se prendre en charge, à être proactif et à s'engager dans le processus de transformation individuelle et familiale pour faire face à la situation de stress, lui donner un sens constructif et permettre à chacun de trouver un nouvel équilibre pour lui-même afin de participer au renouvellement du bien-être familial (Pelchat et Lefebvre, 2004).

La transformation individuelle et familiale découle de l'utilisation de comportements résilients permettant de s'adapter à l'événement traumatisant, le TCC. Ce processus adaptatif permet non seulement un retour à l'équilibre, mais aussi un gain significatif en termes de développement de nouvelles compétences et habiletés et de modification des croyances et des valeurs (Man, 2002). Avec la résilience, un sens positif est accordé à l'événement traumatisant (Pelchat et al., 2002). L'interprétation positive de la situation de santé est considérée comme la stratégie d'adaptation la plus efficace pour résorber le traumatisme psychologique laissé par le TCC d'un membre de la famille. Trois voies permettent de construire un sens positif à l'événement: celle menant à une raison d'être (mission), celle amenant la découverte de gains et de bénéfices a posteriori (apprentissages) et celle caractérisée, dès le départ, par un sens positif (Pelchat et al., 2002). La transformation résulte des comportements résilients des membres de la famille, leur permet de rebondir, de se reconstruire et de se donner les moyens de se projeter dans l'avenir malgré l'événement éprouvant (Man, 2002; Pelchat et al., 2002); Pelchat et al., 2001).

Cependant, le soutien est essentiel pour permettre aux personnes et aux familles de cheminer dans cette épreuve, pour découvrir et mettre en œuvre des comportements et des attitudes de résilience (Girgis et al., 1999; Kim et Alvi, 1999; Girgis et Sanson-Fisher, 1998; Pelchat, 1994) et pour garder un projet de vie satisfaisant dans la circonstance (Cyrulnik, 2002; Pelchat et al., 2002; Fisher, 1994). Concrètement, comment soutenir les familles dans leur expérience?

Le développement et l'actualisation de l'interdisciplinarité et du partenariat dans les relations avec les personnes et les familles, au sein des équipes de soins et entre les établissements ont pris beaucoup d'importance au cours des dernières années. Cependant, il n'en demeure pas moins que la relation entre les acteurs demeure parfois une source de malaise et d'insatisfaction. Les personnes et les familles sont désappointées par le peu d'information qui leur est transmise et par le manque de soutien qui leur est offert. De leur côté, les professionnels vivent parfois de la frustration du fait de ne pas pouvoir répondre aux questions des personnes et des familles et sont souvent pris de court devant leurs réactions et leurs émotions imprévisibles. Les familles et les professionnels vivent, chacun de leur côté, des sentiments d'impuissance et de perte de contrôle. Le partenariat est une stratégie intéressante pour faire face aux sentiments d'impuissance et de perte de contrôle (Man, 2002). Le partenariat permet aux familles de prendre le contrôle sur leur vie par le biais d'expériences positives. Il constitue aussi une ressource pour les professionnels pour faire face aux sentiments d'impuissance et de malaise vécus dans leurs relations avec les familles.

La recherche et l'expérience clinique montrent qu'une relation reposant sur le partage réciproque des savoirs et des expertises dans un rapport égalitaire est garante d'une relation satisfaisante, malgré une atmosphère chargée d'émotions (Pelchat et al., 2001 ; Pelchat et al., 2000; Bouchard, 1999; Horowitz, Horowitz, Orsini, Antoine et Hill, 1998; Lucyshyn, Noxon, Glang et Cooley, 1996; Pelchat, 1995). Les professionnels analysent la situation de santé de la personne à travers leur savoir disciplinaire et leur expérience personnelle et professionnelle et partagent leurs constats avec la personne affectée et sa famille. Ces dernières analysent la situation de santé à travers leur savoir particulier et leur expérience de leur situation de vie, qu'elles confrontent au savoir d'expert des professionnels afin de mieux comprendre le TCC.

L'équipe de soins utilise ses savoirs d'experts pour aider la personne et la famille à modifier une situation difficile tout en reconnaissant les connaissances et les compétences de celles-ci à trouver des solutions, à les mettre en œuvre et à les évaluer (Sohlberg, McLaughlin, Todis, Larsen et Glang, 2001; Lucyshyn et al., 1996). Pour ce faire, il doit y avoir un échange mutuel dans une relation égalitaire entre tous les acteurs. Il s'agit là de savoirs 
complémentaires qui, ensemble, forment un savoir commun (Pelchat, 2001; Pelchat et al., 2001; Bouchard, 1999). C'est cette mise en commun qui assure le succès des relations, de la réadaptation et, par extension, de l'adaptation individuelle et familiale à la situation de vie du TCC (Solhberg et al., 2001; Horowitz et al., 1998). D'ailleurs, dans une perspective systémique, il est généralement reconnu que la famille constitue le principal et le plus important réseau de soutien auprès de la personne ayant subi un TCC (Blaise, 1997; Krier, 1997; Madinier, 1997). Cette reconnaissance amène de plus en plus de professionnels à travailler en étroite collaboration avec la famille et son réseau de soutien, dans une perspective de partenariat (Bouchard, 1999; Pelchat et Berthiaume, 1996).

Dans le contexte des relations personnefamille-équipe de soins, le partenariat implique un savoir-être et un savoir-faire qui se manifestent notamment dans l'attitude du professionnel. Au moment des entretiens, le professionnel valide ce que la personne et la famille connaissent du problème de santé, de leurs expériences antérieures au sujet du problème, de leurs valeurs, croyances et préjugés. Il est important de vérifier ce que la famille sait déjà à propos de la situation, car cela permet d'ajuster le message à la situation particulière de cette famille. Dans la relation, le professionnel favorise le partage mutuel des perceptions dans le respect de chacun montrant ainsi à chaque membre de la famille qu'il le comprend. Il reconnaît les compétences de la personne et de la famille et investit ses forces afin de favoriser son implication dans le traitement à venir. Cela favorise la collaboration de la personne et de la famille, le développement de comportements résilients, car elles comprennent mieux la situation et se sentent en confiance avec le professionnel. Au cours de l'entretien, des stratégies visant à soutenir les forces de la personne et de la famille sont utilisées et suppléent aux difficultés rencontrées tant au plan des connaissances qu'au plan émotif. En d'autres mots, il s'agit de partir de la culture de la famille et de traduire l'information en conséquence. Le fait de s'enquérir de l'histoire de la personne et de la famille permet au professionnel de connaître les forces et les habiletés de celles-ci. La connaissance mutuelle laisse alors émerger la reconnaissance de l'expertise et des savoirs de chacun. Il s'agit là du plus grand défi du partenariat, mais aussi de l'enjeu le plus important pour la satisfaction de tous les acteurs. L'expertise des personnes et des familles doit être connue, reconnue et renforcée. Concrètement, il s'agit d'apprendre à les écouter et à les entendre, à solliciter le partage de leur expertise, à ne pas apporter de solutions toutes faites, mais plutôt à les amener à trouver eux-mêmes leurs propres solutions.

Le caractère soudain et inattendu du traumatisme craniocérébral de même que l'incertitude qui enveloppe son diagnostic et son pronostic impliquent que certaines phases du processus de deuil peuvent se prolonger dans le temps. Cela fait en sorte que cet événement est psychologiquement et émotivement très souffrant pour les familles. Le processus de deuil présenté ici s'apparente à celui vécu par des familles lors d'autres problématiques de santé et n'est pas exclusif au traumatisme craniocérébral.

Apprendre qu'un membre de la famille a eu un traumatisme craniocérébral ou est atteint d'une maladie dégénérative ou incurable est un événement pénible et marquant pour la personne affectée et pour sa famille, mais aussi un moment difficile pour les professionnels. Toutefois, si les professionnels réfléchissent sur leur façon de vivre leurs propres deuils, s'ils ont conscience de ce qui se passe et comprennent ce que les familles vivent, ils pourront développer une manière de dire les choses et de les soutenir qui sera une force dans leurs relations avec elles plutôt qu'une contrainte. Ils contribueront ainsi à soutenir la résilience des familles et leur mieux-être.

\section{BIBLIOGRAPHIE}

BLAISE, J.L. (1997). «Traumatisme crânien: blessure sociale», dans Handicaps et inadaptations. Les cahiers du CTNERHI, 75-76, p. 5-20.

BOLDUC, M. (1996). Perceptions des besoins des familles dont un des membres, victime d'une traumatisme crânien, est aux soins intensifs, Mémoire de maîtrise (non publié), québec, Université Laval.

BOUCHARD, J.M. (1999). «Famille et savoirs à partager: des intentions à l'action ", Apprentissage et socialisation, 19(2), p. 47-57.

BOSS, P. (1999). Ambiguous Loss, Cambridge, Harvard University Press.

BRIÈRE, N. (1996). Interventions infirmières auprès des familles ayant un membre hospitalisé à une unité de traumatologie, Mémoire de maîtrise (non publié), Université de Montréal, Montréal.

BRZUSTOWSKI, M. (2001). L'annonce $d u$ handicap au grand accidenté, France, Éres.

CAMPBELL, E.M., et R.W. SANSONFISHER (1998). "Breaking bad news 3: encouraging the adoption of best practices", Behavioral Medecine, 24(2), p. 73-80.

COHADON, F. (2000). Sortir du coma, Paris, Odile Jacob.

COHEN, M.H. (1993). "The unknown and the unknownable. Managing sustained uncertainty », Western Journal of Nursing Research, 15(1), p. 77-96.
COLANTONIO, A., G. RATCLIFF, S. CHASE, S. KELSEY, M. ESCOBAR et L. VERNICH (2004). «Long-term outcome after moderate to severe traumatic brain injury ", Disability \& Rehabilitation, 26(5), p. 253-261.

CYRULNIK, B. (2002). Un merveilleux malheur, Paris, Odile Jacob.

DAWSON, D.R. et M. CHIPMAN (1995). "The disablement experienced by traumatically brain-injured adults living in the community », Brain Injury, 9(4), p. 339-353.

DELL ORTO, A.E. et P.W. POWER (2000). Head Injury and the Family. A Life Living Perspective, Boca Raton, Gr/St. Lucie Press.

DENORMANDIE, P. et E. HIRSCH (1999). Espace éthique. Mission Handicaps. L'annonce du handicap, Assistance hôpitaux publique de Paris, Paris.

DOKA, J.C.L. (1989). Disenfranchised grief: Recognizing Hidden Sorrow, Toronto, Lexington Books.

ERGH, T.C., R.A. HANKS, L.J. RAPPORT et R.D. COLEMAN (2003). "Social support moderates caregiver satisfaction with life following TBI », The Journal of Clinical and Experimental Neuropsychology, 25(8), p. 1090-1101.

ERGH, T.E., L.J. RAPPORT, R.D. COLEMAN et R.A. HANKS (2002). "Predictors of distress among caregivers of patients with traumatic brain injury", Journal of Head Trauma Rehabilitation, 17(2), p. 155-174.

FALLOWFIELD, L. et V. JENKINS (2004). "Communicating sad, bad, and difficult news in medicine», The Lancet, 363, p. 312-319.

FISHER, G.N. (1994). Le ressort invisible. Vivre l'extrême, Paris, Seuil.

FONDATION POUR L'ENFANCE (2001). La résilience: le réalisme de l'espérance, Ramonville, Érès.

FRÉNISY, M.C., H. BÉHONY, M. FREYSZ, D. HONNART, J.P. DIDIER et J.L. SAUTREAUX (1999). "À propos d'une expérience d'accompagnement psychologique des familles de traumatises crâniens ", Annales médico-psychologiques, 157(3), p. 159-165.

GÉLINAS, I. (1998). "Réflexion sur les déterminants de l'adaptation des familles de personnes traumatisées cranio-cérébrales", dans J.-C. KALUBI, B. MICHALLET, N. KORNER-BITENSKY et S. TÉTREAULT (dir.), Innovations, apprentissages \&ै réadaptation en déficience physique, Montréal, Isabelle Quentin Éditeur.

GIRGIS, A., R.W. SANSON-FISHER et M.J. SCHOFIELD (1999). "Is there consensus between breast cancer patients and providers on guidelines for breaking bad news? », Behavioral Medicine, 25(2), p. 69-77.

GIRGIS, A. et R.W. SANSON-FISHER (1998). «Breaking bad news 1: current best advice for clinicians », Behavioral Medicine, 24, p. 53-59.

HOROWITZ, R.C., S.H. HOROWITZ, J. ORSINI, R.L. ANTOINE et D.M. HILL (1998). "Including families in collaborative care», Families Systems Health, 16, p. 71-83. 
JACOBS, H.E. (1988). «The Los Angeles head injury survey: Procedures and initial findings ", Archives of Physical Medicine and Rehabilitation, 69, p. 425-431.

KALUBI, J.C., B. MICHALLET, J.M. BOUCHARD et D. PELCHAT (1998). "Des silences et des maux: regard sur les perceptions de parents et de médecins suite à l'annonce de la déficience motrice cérébrale», dans "Groupe inter-réseaux de recherche sur l'adaptation de la famille et de son environnement et Centre de réadaptation Le Bouclier ", Innovations, apprentissages \& réadaptation en déficience physique, Montréal, Isabelle Quentin Éditeur.

KIM, M.K. et A. ALVI (1999). «Breaking the bad news of cancer: the patient perspective», Laryngoscope, 109(7-1), p. 1064-1067.

KOSKINEN, S. (1998). "Quality of life 10 years after a very severe traumatic brain injury: the perspective of the injured and the closest relative», Brain Injury, 12(8), p. 631-648.

KRIER, M. (1997). «Les familles de traumatisés crâniens graves: actions et représentations", Handicaps et inadaptations - Les cahiers du CTNERHI, 75-76, p. 33-48.

KÜBLER-ROSS, E. (1981). Living with Death and Dying, New York, Macmillan.

LACROIX, A. et J.P. ASSAL (2003). L'éducation thérapeutique du patient, Paris, Maloine.

LEACH, L. R., D.E. FRANK, D.E. BOUMAN et J. FARMER (1994). "Family functioning, social support and depression after traumatic brain injury», Brain Injury, 8, p. 599-606.

LEFEBVRE, H., D. PELCHAT et M.J. LEVERT (à paraître). "L'annonce du traumatisme craniocérébral et des incapacités », Sciences sociales et santé.

LEFEBVRE, H., D. PELCHAT, J.C. KALUBI et B. MICHALLET (2002). "Expérience distinctive de parents, de professionnels et de médecins lors de l'annonce de la déficience motrice cérébrale », dans S. TÉTREAULT, P. BEAUPRÉ, J.-C. KALUBI, et B. MICHALLET (dir.), Famille et situation de handicap. Comprendre pour mieux intervenir, Sherbrooke, Éditions du CRP.

LEFEBVRE, H., D. PELCHAT, B. SWAINE, I. GÉLINAS et M.J. LEVERT (2004). Le traumatisme craniocérébral suite à un accident de la route: les mots des personnes, des familles, des médecins et des professionnels. Recherche en soins infirmiers, 78, 14-34.

LEFEBVRE, H., M. VANIER, B. SWAINE, E. DUTIL, M. PÉPIN, P. FOUGEYROLLAS, C. RAINVILLE, M.A. MCCOLL, C. DUMONT, D. PELCHAT, B. MICHALLET, I. GÉLINAS, S. DENIS, M. MORIN, H. TROTTIER et M.J. LEVERT (2004). La participation sociale à long terme de personnes ayant subi un traumatisme crânien et l'impact chez les proches, 10 ans post-traumatisme, Rapport de recherche, Montréal, ÉRIFAM, Université de Montréal.

LUCYSHYN, J.M., C. NIXON, A. GLANG et E. COOLEY (1996). "Comprehensive family support for behaviour change in children with ABI », dans G.H.S. SINGER, A. GLANG, J.M.
WILLIAMS (dir.), Children with Acquired Brain Injury: Educating and Supporting the families, Baltimore, Paul H. Brookes.

MADINIER, P. (1997). "Les familles à l'épreuve du traumatisme crânien ", Handicaps et inadaptations - Les cahiers du CTNERHI, 75-76, p. 21-32.

MAGUIRE, P. (1998). «Breaking bad news», European Journal of Surgical Oncology, 24, p. 188-199.

MAN, D.W.K. (2002). «Familiy caregiver's reactions and coping for persons with brain injury », Brain Injury, 16(12), p. 1025-1037.

MISHEL, M.H. (1997). "Uncertainty in acute illness», Annual Review for Nursing Research, 15, p. 57-80.

NOLTE, M.-L. (2000). Disablement, context, and quality of life after brain injury: Measuring what matters (en ligne), disponible: $<$ www.lib.umi.com>

PATUREAU, É. (1984). Étude des problèmes relationnels famille /blessés /équipe de soins au cours de l'évolution des comas traumatiques graves, thèse de doctorat (non publiée), Université de Bordeaux II, France.

PELCHAT, D. (1989). Programme d'intervention précoce auprès de parents d'un enfant ayant une déficience physique, Thèse de doctorat (non publiée), Université de Montréal, Canada.

PELCHAT, D. (1995). «La famille et la naissance d'un enfant ayant une déficience physique», dans F. DUHAMEL (dir.), La santé et la famille: approche systémique en soins infirmiers, Montréal, Gaëtan Morin.

PELCHAT, D. et M. BERTHIAUME (1996). «Intervention précoce auprès de parents ayant une déficience: un lieu d'apprentissages pour les familles et les intervenants », Apprentissage et Socialisation, 17(1-2), p. $105-117$.

PELCHAT, D. et H. LEFEBVRE (2002). «Deuil, Appropriation de compétences Transformation. L'apport du PRIFAM au soutien de la résilience des familles », Pratiques psychologiques, 1, p. 41-52.

PELCHAT, D. et H. LEFEBVRE (2004). Apprendre ensemble. Le PRIFAM, programme d'intervention interdisciplinaire et familiale, Montréal, Chenelière et McGraw-Hill.

PELCHAT, D., H. LEFEBVRE, J.M. BOUCHARD, J.C. KALUBI, B. MICHALLET et S. ROUSSEAU (2000). Processus de l'annonce de la déficience motrice cérébrale: expérience des parents, des professionnels et des médecins, Rapport de recherche, Montréal, ÉRIFAM, Université de Montréal.

PELCHAT, D., H. LEFEBVRE et C. DAMIANI (2002). «Deuil-Appropriation de compétences - Transformation », Pratiques psychologiques, 1, p. 41-52.

PELCHAT, D., H. LEFEBVRE et J.M. BOUCHARD (2001). "L'annonce de la déficience motrice cérébrale: une relation de confiance à construire entre les parents, les professionnels et les médecins», Paediatrics \& Child Health, 6(6),p. 365-374.

PELCHAT, D., H. LEFEBVRE, M. PROULX et J.M. BOUCHARD (2001). «Le partenariat: contextes d'apprentissage», dans J.-C. KALUBI., J.M. BOUCHARD, J.P. POURTOIS et D. PELCHAT (dir.), Partenariat, coopération et appropriation des savoirs, Sherbrooke, Éditions CRP.

PTACEK, J.T. et N.M. ALLISON (2000). "Healthcare providers' perspectives on breaking bad news to patients », Critical Care Nursing Quarterly, 23(2), p. 51-59.

RANDO, T.A. (1986). Loss and Anticipatory Grief, Toronto, Lexington Books.

RAPHAËL, B. (1983). The Anatomy of Bereavement, New York, Basic Books.

SAMPALIS, J.S., R. DENIS, P. FRÉCHETTE, R. BROWN, D. FLEISZER et D. MULDER (1997). «Direct transport to tertiary trauma centers versus transfer from lower level facilities: impact on mortality and morbidity among patients with major trauma», The Journal of Trauma: Injury, Infection, and Critical Care, 43(2), p. 288-296.

SAMPALIS, J.S., A. LAVOIE, S. BOUKAS, H. TAMIM, A. NIKOLIS, P. FRÉCHETTE, R. BROWN, D. FLEISZER, R. DENIS, E. BERGERON, et D. MULDER (1995). «Trauma center designation: initial impact on trauma-related mortality ", The Journal of Trauma, 39(2), p. 232-239.

SCHUMACKER, K.L. et A.I. MELEIS (1995). "Transitions: a central concept in nursing», IMAGE, Journal of Nursing Scholarship, 26(2), p. $72-80$.

SHANFIELD, S.B. et B.J. SWAIN (1984). «Death of adult children in traffic accidents ", Journal of Nervous and Mental Disease, 72(9), p. 533-538.

SHAW, L.R., F. CHAN et C.S. LAM (1997). «Development and application of the Family Involvement Questionnaire in brain injury rehabilitation», Brain Injury, 11(3), p. 219231.

SOCIÉTÉ DE L'ASSURANCE AUTOMOBILE DU QUÉBEC (SAAQ) (2001). Bilan routier 2001 (en ligne), disponible, <ww.saaq. gouv.ca $>$.

SOHLBERG, M.M., K.A. MCLAUGHLIN, B. TODIS, J. LARSEN et A. GLANG (2001). "What does it take to collaborate with families affected by brain injury? A preliminary model», The Journal of Head Trauma Rehabilitation, 16(5), p. 498-511.

STATISTIQUE CANADA (1999). Rapport statistique sur la santé de la population canadienne, Catalogue $\mathrm{n}^{\circ}$ 82-570-XIF.

SAINT-CHARLES, D. (1998). «La reconstruction identitaire des "proches-soignants" des personnes traumatisées craniocérébrales", dans J.C. KALUBI, B. MICHALLET, N. KORNER-BITENSKY et S. TÉTREAULT (dir.), Innovations, apprentissages et réadaptation en déficience physique. Innovations, apprentissages et réadaptation en déficience physique, Montréal, Isabelle Quentin Éditeur.

YANKO, J.R. et K. MITCHO (2001). «Acute care management of severe traumatic brain injuries », Critical Care Nursing Quarterly, 23(4), p. 1-23. 